ARTICLE

https://doi.org/10.1038/s41467-019-12268-5

\title{
Integration of mesopores and crystal defects in metal-organic frameworks via templated electrosynthesis
}

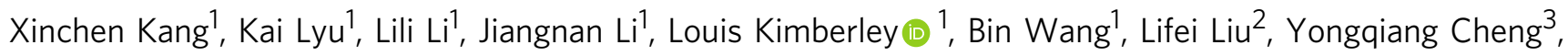

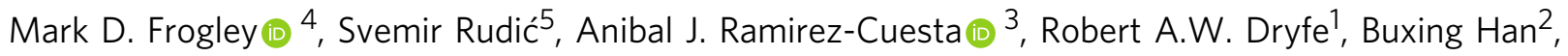
Sihai Yang (1) ${ }^{1} \&$ Martin Schröder (iD ${ }^{1}$

Incorporation of mesopores and active sites into metal-organic framework (MOF) materials to uncover new efficient catalysts is a highly desirable but challenging task. We report the first example of a mesoporous MOF obtained by templated electrosynthesis using an ionic liquid as both electrolyte and template. The mesoporous Cu(II)-MOF MFM-100 has been synthesised in 100 seconds at room temperature, and this material incorporates crystal defects with uncoupled $\mathrm{Cu}(\mathrm{II})$ centres as evidenced by confocal fluorescence microscopy and electron paramagnetic resonance spectroscopy. MFM-100 prepared in this way shows exceptional catalytic activity for the aerobic oxidation of alcohols to produce aldehydes in near quantitative yield and selectivity under mild conditions, as well as having excellent stability and reusability over repeated cycles. The catalyst-substrate binding interactions have been probed by inelastic neutron scattering. This study offers a simple strategy to create mesopores and active sites simultaneously via electrochemical formation of crystal defects to promote efficient catalysis using MOFs.

\footnotetext{
${ }^{1}$ School of Chemistry, University of Manchester, Manchester M13 9PL, UK. ${ }^{2}$ Beijing National Laboratory for Molecular Sciences, CAS Key Laboratory of Colloid, Interface and Chemical Thermodynamics, Institute of Chemistry, Chinese Academy of Science, Beijing 100190, China. ${ }^{3}$ Oak Ridge National Laboratory, Oak Ridge, TN 37831, USA. ${ }^{4}$ Diamond Light Source, Harwell Science Campus, Oxfordshire OX11 ODE, UK. ${ }^{5}$ ISIS Facility, STFC Rutherford Appleton Laboratory, Oxfordshire OX11 OQX, UK. Correspondence and requests for materials should be addressed to S.Y. (email: Sihai.Yang@manchester.ac.uk) or to M.S. (email: M.Schroder@manchester.ac.uk)
} 
T he application of metal-organic framework (MOF) materials in catalysis is often restricted by the hindered mass transport of substrates through micropores and the lack of accessible active sites ${ }^{1-5}$. Significant efforts have been devoted to the synthesis of mesoporous MOFs to facilitate substrate transport for catalysis $^{6,7}$, and creating crystal defects to serve as active sites ${ }^{8-10}$. The most common approach to create mesopores in MOFs is to construct the framework using elongated ligands and/or large metal clusters via isoreticular chemistry ${ }^{11,12}$. However, the synthesis of extended organic linkers usually involves multi-step reactions, and the resultant MOFs often suffer from limited framework stability on desolvation. Recently, a number of templating methods using supercritical $\mathrm{CO}_{2}$, ionic liquids (ILs), and surfactants have been reported for the synthesis of mesoporous MOFs with improved mass transport and catalytic properties ${ }^{13-15}$.

Removal of coordinated solvent molecules (typically water) in MOFs can generate open metal sites as Lewis acids for catalysis ${ }^{16,17}$. However, this strategy has a number of limitations. Many MOF systems are built from metal centres with saturated coordination environments and without coordinated labile solvents. Also, the removal of coordinated solvents from metal centres can have a detrimental effect on the overall stability of the framework in activated MOFs, and generated open metal sites are readily saturated by solvent molecules used in catalytic reactions, thus remaining inaccessible to target substrates. Recently, the introduction of crystal defects into MOFs as active sites has given rise to enhanced gas adsorption and catalytic properties ${ }^{18}$. In general, defects in MOFs can be divided into inherent and intentional defects ${ }^{19}$. The former are formed without targeted design by stacking faults or dislocations as crystals grow ${ }^{20}$, while judicious choice of synthetic conditions, such as choice of temperature, $\mathrm{pH}$, pressure and solvents, can promote the generation of defects during the MOF synthesis ${ }^{21,22}$. Defects can thus be produced intentionally during synthesis or via post-synthetic treatment. For example, acid- or base-treatment, solvent exchange and activation have been shown to create random crystal defects ${ }^{23,24}$. However, few processes offer effective control of the formation of crystal defects in MOFs, and, moreover, the characterisation of crystal defects is difficult to achieve. Integration of mesopores and active sites within crystal defects in MOFs via readily scalable synthetic routes could in principle greatly facilitate their applications in catalysis. In addition, electrosynthesis can be used for materials production at varying scales 25,26 .

Here, we describe the templated electrosynthesis of samples of MFM-100 containing crystal defects. Mesoporous MFM-100 has been successfully obtained by rapid $(<100 \mathrm{~s})$ electrosynthesis at room temperature. The IL, 1-octyl-3-methylimidazolium tetrafluoroborate $\left(\mathrm{OmimBF}_{4}\right.$, Supplementary Fig. 1) was used as an electrolyte and it also serves as a template via self-aggregation to create mesopores in the resultant MOF. The structures, morphology and porosity of MFM-100 can be readily controlled by choice of energy input and IL in the synthesis. These MFM-100 materials contain crystal defects [e.g., uncoupled $\mathrm{Cu}$ (II) centres as Lewis acid sites], which have been characterised by confocal fluorescence microscopy (CFM), inelastic neutron scattering (INS), electron paramagnetic resonance (EPR) and Fourier-transform infrared (FTIR) spectroscopy. Significantly, the integration of mesopores and active sites within the MFM-100 scaffold has endowed the resultant MOF with exceptional activity for the aerobic oxidation of a range of alcohols to aldehydes in high yield and selectivity. The MFM-100 catalysts also exhibit excellent stability and reusability.

\section{Results}

Electrosynthesis of MFM-100. Four samples (denoted as a-d) of MFM-100 (also known as NOTT-10027,28) have been synthesised.
MFM-100a was synthesised in a solvothermal reaction using $\mathrm{Cu}$ $\left(\mathrm{NO}_{3}\right)_{2}$ and biphenyl-3,3',5,5'-tetracarboxylic acid $\left(\mathrm{H}_{4} \mathrm{~L}\right.$; Supplementary Fig. 2) in a mixture of DMF/1,4-dioxane/water (2:1:1 v/ $\mathrm{v} / \mathrm{v}$ ) at $80^{\circ} \mathrm{C}$ for 3 days ${ }^{27,28}$ and isolated as single crystals (yield $\sim 75 \%$ ) of well-defined morphology (particle sizes $\sim 3-5$ microns, Fig. 1a). MFM-100(b,c,d) were obtained by electrosynthesis (Fig. 1b-d) in the same solvent mixture as above using copper foil as both cathode and anode with $\mathrm{OmimBF}_{4}$ as the supporting electrolyte (Fig. 1e). During the electrosynthesis, the copper foil at the anode is oxidised to $\mathrm{Cu}(\mathrm{II})$ ions, and simultaneously protons from the ligand are reduced to $\mathrm{H}_{2}$ under the applied potential, affording deprotonated $\mathrm{L}^{4-}$ species that coordinate to $\mathrm{Cu}(\mathrm{II})$ ions in the electrolyte to promote the formation of MFM-100. It is worth noting that water reduction is a side-reaction in this process.

We sought to study the effects of temperature and the concentration of IL on the electrosynthesis of MFM-100. MFM$100 \mathrm{~b}$ was synthesised at $80^{\circ} \mathrm{C}$ with $1 \mathrm{wt} \%$ of IL in the solvent and isolated as a microcrystalline powder with an average particle size of 1.3 microns (Fig. 1b). MFM-100c and MFM-100d were synthesised using 10 and $50 \mathrm{wt} \% \mathrm{IL}$, respectively, in solution at room temperature $\left(25^{\circ} \mathrm{C}\right)$ and were isolated as blue powders of much smaller particle size (average size of 800 and $400 \mathrm{~nm}$ for MFM-100c and MFM-100d, respectively) and irregular morphology (Fig. 1c, d). In comparison to MFM-100c, the increased amount of IL in the synthesis of MFM-100d resulted in reduced particle size, presumably because IL can act as a protecting agent and mediate nucleation during crystal growth. The size distributions of these different MOF particles have been obtained by SEM/TEM measurements (Fig. If and Supplementary Fig. 3). It is worth noting that no precipitate was formed in the synthesis of MFM- 100 at $25^{\circ} \mathrm{C}$ in the absence of an applied voltage over $72 \mathrm{~h}$. Interestingly, the electrosynthesis of MFM-100(b, c, d) can be achieved within $100 \mathrm{~s}$ in a yield of $\sim 30-40 \%$. A yield of $>90 \%$ can be achieved at $500 \mathrm{~s}$ at room temperature for MFM-100c and MFM-100d, offering a highly efficient and readily scalable route for MOF production.

Structural analysis and characterisation of MFM-100. The crystal structure of MFM-100 is shown in Supplementary Fig. 4. Analysis of the samples by powder X-ray diffraction (PXRD) confirms that all Bragg peaks observed correspond to MFM-100 (Fig. 2a) ${ }^{27}$. MFM-100(a,b) are more crystalline than MFM-100(c, d) suggesting that the latter have substantial crystal defects, leading to reduction in long-range order of the solid-state lattice. Vibrational spectroscopy is a commonly used technique to characterise crystal defects ${ }^{19}$. Both INS and FTIR spectroscopy confirm full retention of vibrational features in the electrosynthesised samples of MFM-100 (Fig. 2b, c). The INS features of MFM-100c and MFM-100d are heavily convoluted and broadened, and FTIR spectra show notable decrease in intensity and increase of bandwidth on going from MFM-100a to MFM-100d. These results are consistent with the reduced crystallinity and presence of crystal defects in MFM-100(c,d). A small increase in intensity at $1574 \mathrm{~cm}^{-1}$ (assigned to the $\mathrm{C}=\mathrm{C}$ vibration of the imidazole ring; Supplementary Fig. 5) is observed in the FTIR spectra of MFM-100c and MFM-100d, indicating the presence of trace IL cations, which has been confirmed also by elemental analytical data (Supplementary Table 1). Neither B nor F was detected by ICP-OES or X-ray photoelectron spectroscopy (XPS) analysis, suggesting the absence of IL anions in the framework. Measurements of the contact angle at the MOF to water interface confirm $32.5^{\circ}, 35.9^{\circ}, 46.4^{\circ}$ and $55.4^{\circ}$ for samples MFM$100(a, b, c, d)$, respectively, consistent with the presence of the $\mathrm{Omim}^{+}$cations in the electrosynthesised samples leading to 
a

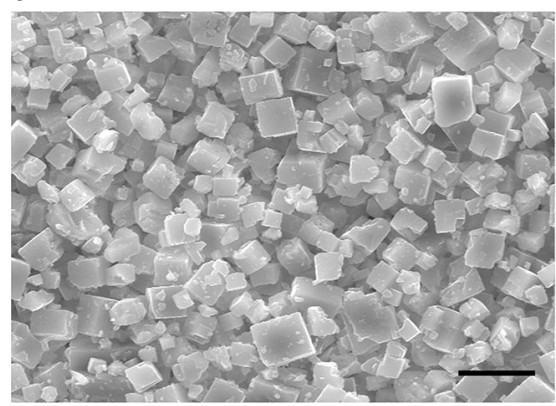

C

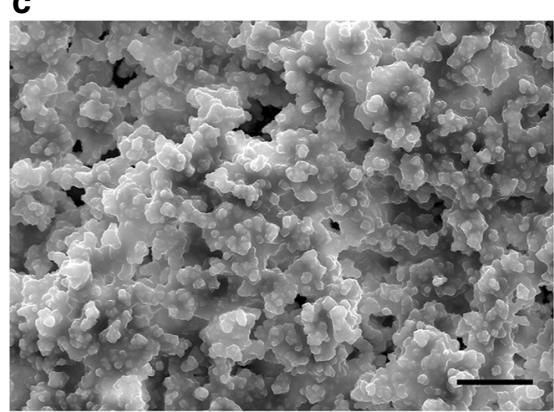

b

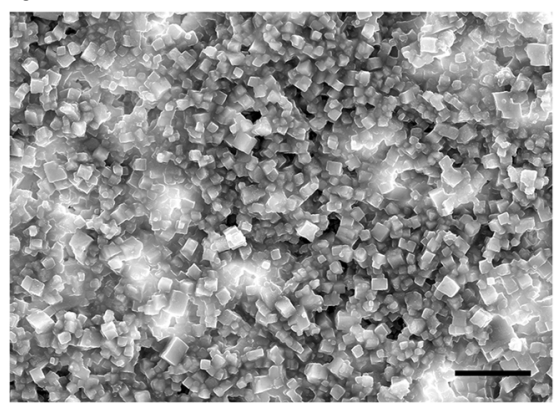

d

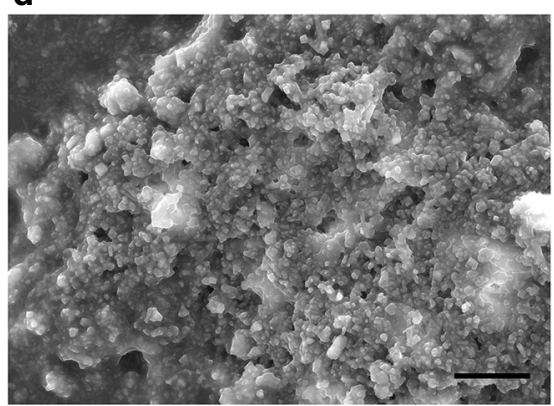

e

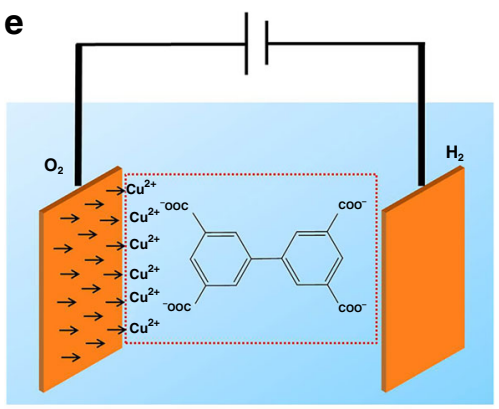

Copper foil Electrolyte

f

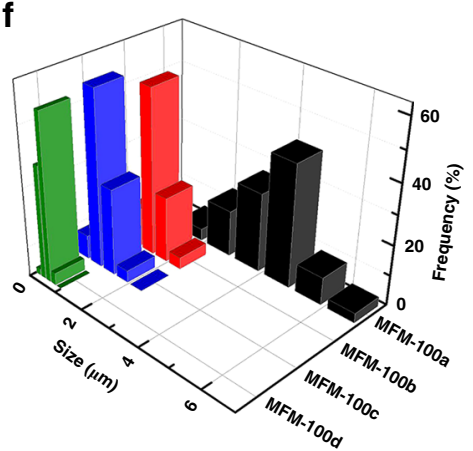

Fig. 1 SEM images and particle size distributions of MFM-100 samples. SEM image of MFM-100a (a), MFM-100b (b), MFM-100c (c) and MFM-100d (d). e Schematic diagram for the electrosynthesis of MFM-100. f Particle size distributions for samples of MFM-100. The scale bar in all SEM images is $10 \mu \mathrm{m}$. The diameter of IL aggregates is $4 \mathrm{~nm}$
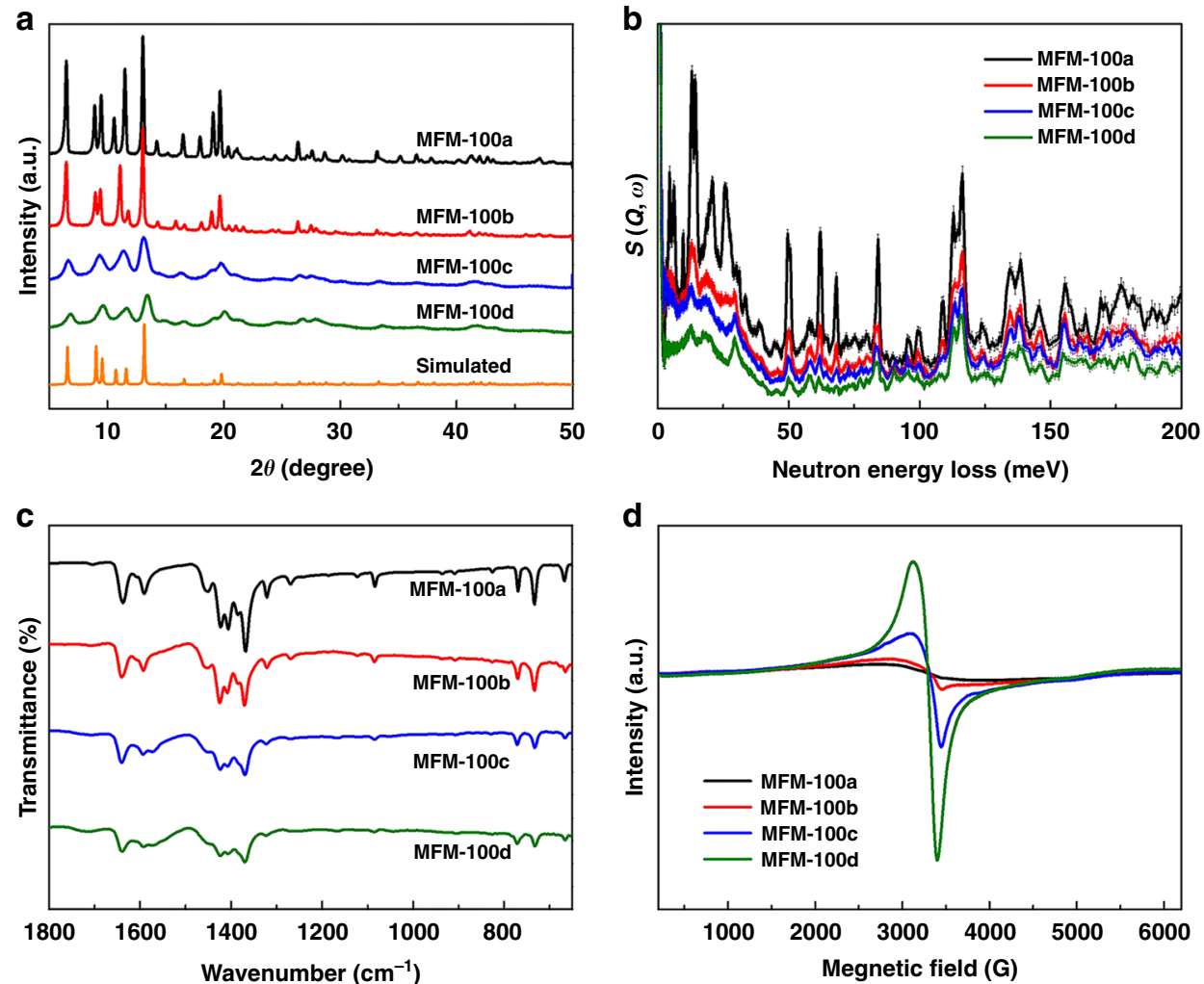

Fig. 2 Characterisation of samples of MFM-100. Comparisons of a PXRD patterns; $\mathbf{b}$ INS spectra; c FTIR spectra; $\mathbf{d}$ solid-state EPR spectra at same sensitivity and concentration of MOF material 
a

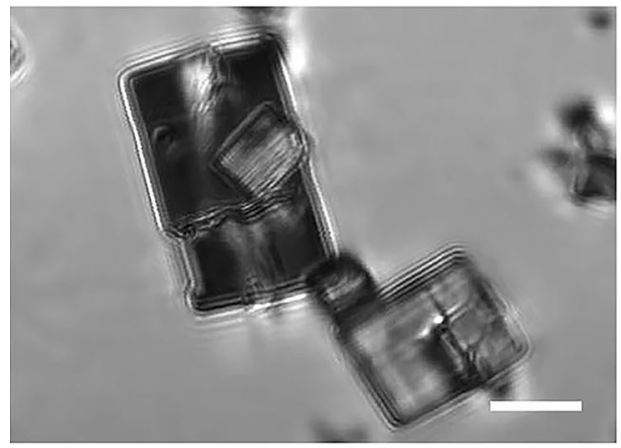

C

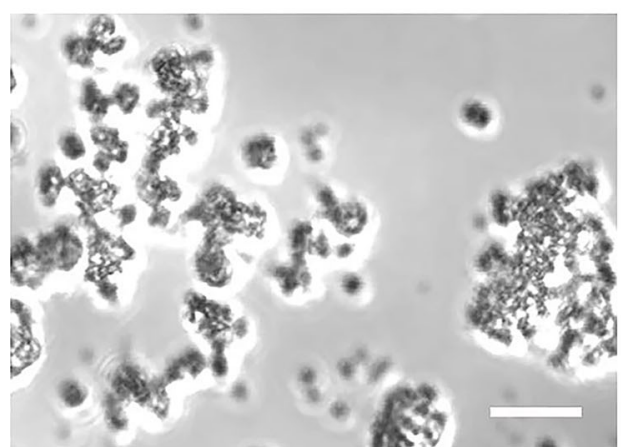

b

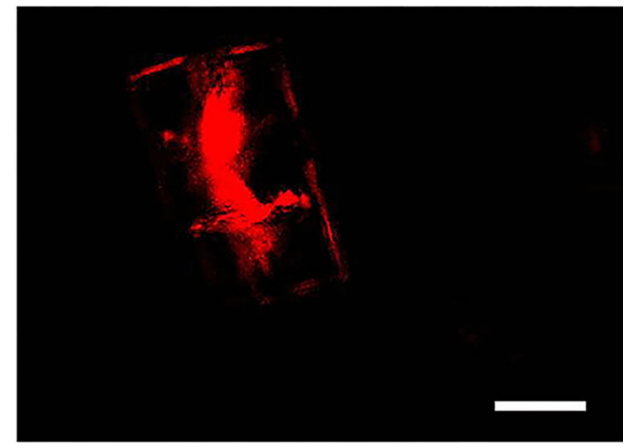

d

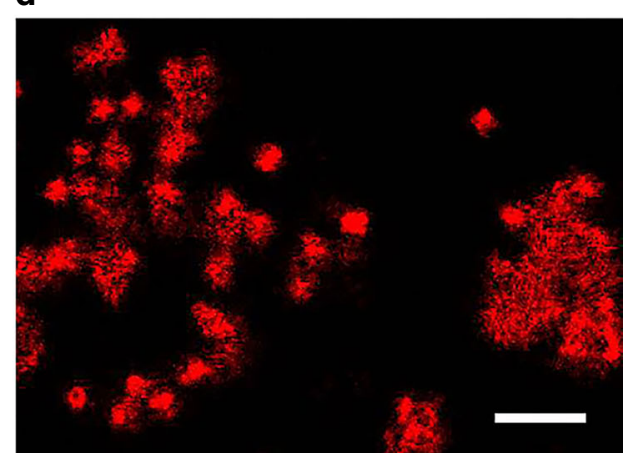

Fig. 3 Micrographs and CFM images. Views of MFM-100a a micrograph, $\mathbf{b}$ CFM and of MFM-100d $\mathbf{c}$ micrograph, $\mathbf{d}$ CFM. The scale bar is $5 \mu \mathrm{m}$ in all images. The fluorescence (shown in red colour) indicates the presence of crystal defects determined by the oligomerisation of furfuryl alcohol

increased hydrophobicity, which is a highly desirable feature to improve the stability of water-sensitive MOFs (Supplementary Fig. 6). Full chemical analysis of these materials has revealed that the stoichiometry of MFM-100 (i.e., the metal-to-ligand ratio) decreases on going from MFM-100a (1.99:1) to MFM-100d (1.79:1), consistent with the partial replacement of $\mathrm{Cu}(\mathrm{II})$ by $\mathrm{Omim}^{+}$(Supplementary Table 1). All four MOFs were digested in hydrochloric acid and the molar ratio of $\mathrm{Omim}^{+} / \mathrm{L}^{4-}$ in solution analysed by ${ }^{1} \mathrm{H}$ nuclear magnetic resonance (NMR) spectroscopy (Supplementary Table 1). This affords highly consistent results with the chemical analytical data.

The EPR spectrum of binuclear paddlewheel $\mathrm{Cu}(\mathrm{II})-\mathrm{Cu}(\mathrm{II})$ species shows only a low intensity signal due to antiferromagnetic coupling between the metal centres. This is reflected in the EPR spectra of the solid-state samples of MFM100a to MFM-100d (Fig. 2d) in which a progressively enhanced signal centred at $3250 \mathrm{G}$ is observed confirming the presence of notable amounts of uncoupled $\mathrm{Cu}$ (II) centres as defects in the framework of MFM-100c and MFM-100d, with an EPR peak width of $\sim 300 \mathrm{G}^{29}$. Thermogravimetric analysis (TGA) suggests that MFM-100c and MFM-100d have lower decomposition temperatures $\left(300^{\circ} \mathrm{C}\right)$ than MFM-100a $\left(340^{\circ} \mathrm{C}\right)$ and MFM$100 \mathrm{~b}\left(320^{\circ} \mathrm{C}\right)$ (Supplementary Fig. 7$)$, consistent with the presence of incomplete metal-ligand coordination in the defective MOFs. The TGA plots of MFM-100c and MFM-100d further confirm the absence of encapsulated free IL molecules within the MOF pores, consistent with the chemical analysis.

Interestingly, it has been recently reported that the introduction of defects as Lewis acid sites in the MOF lattice can be captured by CFM using an acid-catalysed furfuryl alcohol probe reaction ${ }^{30}$. The microphotographs and CFM images reveal a stark comparison between MFM-100a and MFM-100d (Fig. 3). The CFM images can locate emissive products from the oligomerisation of furfuryl alcohol catalysed by Lewis acid sites. MFM-100a only shows fluorescence response at the crystal boundaries (i.e., at edges and gaps), and the absence of mesopores and internal structural defects in MFM-100a is further confirmed by 3D scan images of a single crystal of MFM-100a at different $\mathrm{Z}$ depths (Supplementary Fig. 9). In contrast, all particles of MFM-100d exhibit strong fluorescence that is distributed evenly across the entire particle, directly confirming the presence of homogenous active $\mathrm{Cu}(\mathrm{II})$ sites as Lewis acid defects within MFM-100d.

Analysis of porosity in MFM-100. The porosity of the MFM-100 samples was studied by $\mathrm{N}_{2}$ isotherms at $77 \mathrm{~K}$ (Table 1 and Supplementary Figs. 10 and 11) and the presence of mesopores analysed by the Barrett-Joyner-Halenda (BJH) model ${ }^{7}$. As expected, the highly crystalline MFM-100(a,b) samples show high surface areas $\left(1507-1586 \mathrm{~m}^{2} \mathrm{~g}^{-1}\right)$ that are consistent with the previously reported values ${ }^{27}$; the pore size distribution is centred at $6.5 \AA$ and there is an absence of mesopores. MFM-100c shows a total Brunauer-Emmett-Teller (BET) surface area of $1486 \mathrm{~m}^{2} \mathrm{~g}^{-1}$ and a small amount of mesopores $\left(V_{\text {meso }}=0.41 \mathrm{~cm}^{3} \mathrm{~g}^{-1}\right.$ and $\mathrm{S}_{\text {meso }} /$ $\left.\mathrm{S}_{\text {total }}=0.27\right)$. In contrast, mesopores centred at $4.7 \mathrm{~nm}$ are observed in MFM-100d $\left(V_{\text {meso }}=1.33 \mathrm{~cm}^{3} \mathrm{~g}^{-1}\right.$ and $S_{\text {meso }} / S_{\text {total }}=$ $0.69)$, which has a total BET surface area of $1544 \mathrm{~m}^{2} \mathrm{~g}^{-1}$. Aggregates of $\mathrm{Omim}^{+}$-based ILs have an average size of $\sim 4 \mathrm{~nm}^{31}$, which matches precisely the dimensions of mesopores in MFM$100(c, d)$, confirming the templating effect of IL in the electrosynthesis. The size of IL aggregates relies on the length of alkyl chain and longer alkyl chains result generally in larger aggregates $^{31}$. OmimBF 4 has thus been selected to match the mesopores in the resultant MOFs. The total surface area is observed to decrease slightly with increase of crystal defects because of the collapse of micropores.

Synthesis of MFM-100 under different conditions. To confirm further the critical role of electrosynthesis and IL in the synthesis of defective and mesoporous MOFs, MFM-100(b'-d') were 
Table 1 Summary of the porosity of all MOFs in this study

\begin{tabular}{|c|c|c|c|c|c|}
\hline Entry & Samples & $S_{\text {total }}\left(m^{2} g^{-1}\right)$ & $S_{\text {meso }}\left(m^{2} g^{-1}\right)$ & $D_{\text {meso }}(\mathrm{nm})$ & $V_{\text {meso }}\left(\mathrm{cm}^{3} \mathrm{~g}^{-1}\right)$ \\
\hline 1 & MFM-100a & 1507 & 142 & - & - \\
\hline 2 & MFM-100b & 1586 & 170 & - & - \\
\hline 3 & MFM-100c & 1486 & 399 & 4.6 & 0.41 \\
\hline 4 & MFM-100d & 1544 & 1069 & 4.7 & 1.33 \\
\hline 5 & HKUST-1a & 1017 & 46.6 & - & - \\
\hline 6 & HKUST-1b & 719 & 581 & 4.9 & 0.71 \\
\hline 7 & MOF-2a & 196 & 214 & 4.3 & 0.23 \\
\hline 8 & MOF-2b & 214 & 236 & 5.1 & 0.31 \\
\hline
\end{tabular}

synthesised using the same conditions as MFM-100(b-d), but in the absence of an applied potential. The solvothermal synthesis was carried out over 3 days to give the samples MFM-100(b'-d'). These materials show improved crystallinity by PXRD and similar morphology and particle size distributions compared with MFM100(b-d) (Supplementary Figs. 12 and 13), indicating that defects were generated primarily by the rapid electrosynthesis. In addition, MFM-100d" was prepared by electrosynthesis at $8 \mathrm{~V}$ for $3 \mathrm{~h}$ in the absence of an IL at room temperature. MFM-100d" shows ordered cubic morphology with highly crystalline structure and there is an absence of mesopores (Supplementary Figs. 14-16), indicating that the IL plays a crucial role in creating defects and mesopores. The effect of potential was also investigated via the synthesis of MFM- $100 \mathrm{~d}_{6 \mathrm{~V}}$ and MFM-100 $10 \mathrm{~V}$ at an applied potential of 6 and $10 \mathrm{~V}$, respectively. Overall, the crystallinity of the resultant MOF increases and the particle size reduces with increasing potential (Supplementary Figs. 17 and 18).

Synthesis and characterisation of selected $\mathrm{Cu}(\mathrm{II})$-based MOFs. The Cu(II)-based MOFs, HKUST-1 and MOF-2 ${ }^{32}$, have also been prepared by solvothermal synthesis (denoted as HKUST-1a and MOF-2a) and by templated electrosynthesis (denoted as HKUST$1 \mathrm{~b}$ and MOF-2b) as described above (see Methods for details). The micropore sizes of HKUST-1 and MOF-2 are 6.8 and $13.1 \AA$, respectively. Analysis of the structure and porosity of these samples confirms that the electrosynthesised MOFs show reduced crystallinity and the presence of significant amounts of mesopores (Table 1 and Supplementary Figs. 19 and 20) compared with the samples obtained by solvothermal synthesis. This suggests that the strategy based upon templated electrosynthesis developed here has general applicability to the synthesis of mesoporous, defective $\mathrm{Cu}(\mathrm{II})$-based MOFs.

Catalytic activity of $\mathrm{Cu}(\mathrm{II})$-based MOFs for alcohol oxidation. Aldehydes are key intermediates and high-value chemicals in the perfume and pharmaceutical industries ${ }^{33,34}$. Oxidation of alcohols over $\mathrm{Cu}$ (II)-based catalysts is an efficient method to synthesise aldehydes ${ }^{7,35-37}$, and we therefore tested the catalytic activity of MFM-100, HKUST-1 and MOF-2 for the oxidation of a range of primary and secondary alcohols using 2,2,6,6-tetramethyl-piperidine-1-oxyl (TEMPO) as a co-catalyst (Table 2). There was no conversion of benzyl alcohol in the absence of MOF catalyst or using IL as the catalyst. Oxidation of benzyl alcohol to benzaldehyde was undertaken using solvothermally prepared catalyst. MFM-100a gave a yield of $40 \%$ and exhibited higher activity than HKUST-1a (yield $=19 \%$ ) and MOF-2a (yield $=$ $34 \%$ ), indicating that MFM-100 is a better candidate for alcohol oxidation. Interestingly, the yield of benzaldehyde increases on going from MFM-100a (40\%) to MFM-100d (>99\%). Similarly, HKUST-1b and MOF-2b show better catalytic performance than
HKUST-1a and MOF-2a. Importantly, MFM-100d shows leading catalytic performance for the oxidation of alcohols compared with a range of reported $\mathrm{Cu}(\mathrm{II})$-MOFs (Supplementary Table 3). The catalytic performance of other samples of MFM-100 is summarised in Supplementary Table 2. It is widely accepted that particle size has a strong influence on the catalytic performance. Compared with MFM-100d, MFM-100d' shows notably reduced catalytic activity (yield $=61 \%$ ). In addition, MFM- $100 \mathrm{~d}_{10 \mathrm{~V}}$, showing smaller particle size than MFM-100d, also gave a reduced yield of benzylaldehyde (90\%). Thus, crystal defects consisting of uncoupled $\mathrm{Cu}(\mathrm{II})$ centres in MFM-100(c,d) play a critical role in the observed catalytic activity, leading to the quantitative conversion of benzyl alcohol with MFM-100d.

To investigate the effect of pore size (and hence mass transport) on catalytic activity, the oxidation of a larger substrate, salicin, was tested over all catalysts. Salicin is widely presented in bark and leaves of various species of plants, and the transformation of salicin into useful chemicals is a promising target in biomass conversion ${ }^{38}$. MFM-100d with an increased amount of mesopores $\left(V_{\text {meso }}=0.39\right.$ and $1.17 \mathrm{~cm}^{3} \mathrm{~g}^{-1}$ for MFM-100c and MFM-100d, respectively) exhibits significantly improved yields of aldehyde (46\% and $>99 \%$ for MFM-100c and MFM-100d, respectively), demonstrating the critical role of mesopores on the transport and hence conversion of bulky substrates. In comparison, HKUST-1a and MOF-2a both show poor activity for this conversion. Interestingly, mesoporous HKUST-1b, which has similar pore size as MFM-100d, shows relatively poor catalytic activity due to fewer defects and more crystalline framework structure (Supplementary Fig. 19a). The strong fluorescence in CFM images originates from the oligomerisation product of furfuryl alcohol, the size of which is similar to that of benzyl alcohol. The homogenous distribution of the fluorescence across the entire MOF crystal indicates that the reaction occurs both within the MOF pores and on the surface of particles.

The oxidation of thiophen-2-ylmethanol and 3,3',5,5' -tetrakis (trifluoromethyl)benzhydrol of distinct molecular sizes $(3.6 \times 6.8$ and $7.0 \times 10.0 \AA^{2}$, respectively) was also studied (Table 2). As before, MFM-100(c,d) exhibit notably higher activity than MFM$100(\mathrm{a}, \mathrm{b})$ and MFM-100d affords quantitative production of the corresponding aldehydes in both cases. The time-dependence of the formation of aldehyde further confirmed the critical effect of pore size on substrate selectivity (Fig. 4a, b). Significantly, although with lower dosage of TEMPO and base, MFM-100d shows higher activity for alcohol oxidation than all previously reported $\mathrm{Cu}(\mathrm{II})-\mathrm{MOFs}^{7,39,40}$. These results confirm that integration of mesopores and uncoupled $\mathrm{Cu}$ (II) sites in MFM-100d results in its exceptional catalytic performance.

The reusability of MFM-100d was studied for the oxidation of benzyl alcohol. The catalyst shows excellent stability over five repeated cycles of reaction without detectable loss of activity (Fig. 4c). To test the possible leaching of any catalytic active sites 
Table 2 Summary of the oxidation of selected alcohols to aldehydes using Cu(II)-MOFs as catalysts

\begin{tabular}{|c|c|c|c|c|c|c|}
\hline Entry & substrates & Size $(\AA)^{\dagger}$ & Products & catalysts ${ }^{\perp}$ & $\begin{array}{l}\text { Time } \\
\text { (h) }\end{array}$ & Yield $(\%)^{\ddagger}$ \\
\hline 1 & & \multirow{8}{*}{$4.3,7.5$} & & MFM-100a & 3 & 40 \\
\hline 2 & & & & MFM-100b & 3 & 51 \\
\hline 3 & & & & MFM-100c & 3 & 87 \\
\hline 4 & & & & MFM-100d & 3 & $>99$ \\
\hline 5 & & & & HKUST-1a & 3 & 19 \\
\hline 6 & & & & HKUST-1b & 3 & 34 \\
\hline 7 & & & & MOF-2a & 3 & 34 \\
\hline 8 & & & & MOF-2b & 3 & 53 \\
\hline 9 & & \multirow{4}{*}{$3.6,6.8$} & & MFM-100a & 5 & 40 \\
\hline 10 & & & & MFM-100b & 5 & 52 \\
\hline 11 & & & & MFM-100c & 5 & 84 \\
\hline 12 & & & & MFM-100d & 5 & $>99$ \\
\hline 13 & & \multirow{8}{*}{$7.0,9.5$} & & MFM-100a & 18 & 16 \\
\hline 14 & & & & MFM-100b & 18 & 22 \\
\hline 15 & & & & MFM-100c & 18 & 46 \\
\hline 16 & & & & MFM-100d & 18 & $>99$ \\
\hline 17 & & & & HKUST-1a & 18 & 12 \\
\hline 18 & & & & HKUST-1b & 18 & 25 \\
\hline 19 & & & & MOF-2a & 18 & 17 \\
\hline 20 & & & & MOF-2b & 18 & 30 \\
\hline 21 & & \multirow{4}{*}{$7.0,10.0$} & & MFM-100a & 16 & 18 \\
\hline 22 & & & & MFM-100b & 16 & 26 \\
\hline 23 & & & & MFM-100c & 16 & 58 \\
\hline 24 & & & & MFM-100d & 16 & $>99$ \\
\hline
\end{tabular}

'Reaction conditions: $0.1 \mathrm{mmol}$ catalyst (based on Cu content from ICP); $1 \mathrm{mmol}$ alcohol, 0.1 equiv TEMPO; 0.2 equiv $\mathrm{K}_{2} \mathrm{CO}_{3} ; 4 \mathrm{~mL}$ solvent (entries $1-12 \mathrm{MeCN}, 13-24 \mathrm{DMF}$ ); 1 atm $\mathrm{O}_{2} ; 75^{\circ} \mathrm{C}$ TKinetic diameter of width and length of different compounds were measured by GaussView software

${ }^{\perp}$ There is no conversion in the absence of MOF catalyst for all substrates

Average yield of aldehyde. All experiments were performed three times

into solution, the catalyst was removed via hot filtration at an alcohol conversion of $50 \%$, and no notable further reaction was found to proceed using the solvate, confirming the absence of leaching of catalytically-active $\mathrm{Cu}$ (II) ions during the reaction. The bound $\mathrm{Omim}^{+}$cations in MFM-100d increases the framework hydrophobicity and leads to high stability in polar solvents such as MeCN and DMF. Used MFM-100d was separated from solution and characterised by SEM, PXRD, FTIR and $\mathrm{N}_{2}$ adsorption/desorption isotherms at $77 \mathrm{~K}$ (Supplementary Fig. 21). Little change was observed compared with that of a fresh sample, demonstrating the excellent stability of MFM-100d for the oxidation of alcohols.

Vibrational analysis of host-guest binding dynamics. INS has been applied to investigate the host-guest binding interaction between MFM-100a and MFM-100d and benzyl alcohol. The excellent agreement between the density functional theory (DFT)calculated and experimental INS spectra for solid benzyl alcohol allowed full assignment of its vibrational modes (Supplementary Fig. 22). Significant increases in the peak intensity of INS spectra of MFM-100(a,d) were observed upon adsorption of benzyl alcohol. Comparison of the difference INS spectrum (i.e., signals for the adsorbed substrate together with changes in the local MOF environment) with that of solid benzyl alcohol revealed a number of changes (Fig. $4 \mathrm{~d})$. The peaks at low energy $(<20 \mathrm{meV})$, assigned to the translational and rotational modes of benzyl alcohol, shift to lower energy with a continuum profile, indicating the adsorbed benzyl alcohol molecules are disordered on the pore surface and have hindered motion owing to the binding to the surface sites. The INS peak at $162 \mathrm{meV}$ (assigned to the wagging mode of the -OH group in benzyl alcohol) almost disappears on adsorption in MFM-100d, indicating deprotonation of the alcohol on the MOF surface. The peaks at 135 and $152 \mathrm{meV}$ (assigned to the wagging and twisting modes of the $-\mathrm{CH}_{2}$ - group, respectively) shift to higher energies consistent with the formation of benzyl alcohol anion on the MOF surface, which leads to its activation and subsequent oxidation.

Summary. We report the use of templated electrosynthesis to integrate mesopores with crystal defects in $\mathrm{Cu}(\mathrm{II})$-based MOFs. This enables the production of MFM-100 within $100 \mathrm{~s}$ at room temperature, and the structures, morphology, porosity and crystal defects of the resultant materials can be readily controlled by the use of ILs as templates in the synthesis. The presence and nature of crystal defects in MFM-100 have been characterised comprehensively by CFM, INS, EPR, FTIR and chemical analysis, and these materials show excellent catalytic activity and stability for the oxidation of a range of alcohols, including inactive primary alcohols. We found that the co-presence of crystal defects and mesopores greatly promotes catalytic oxidation with exceptional 
a

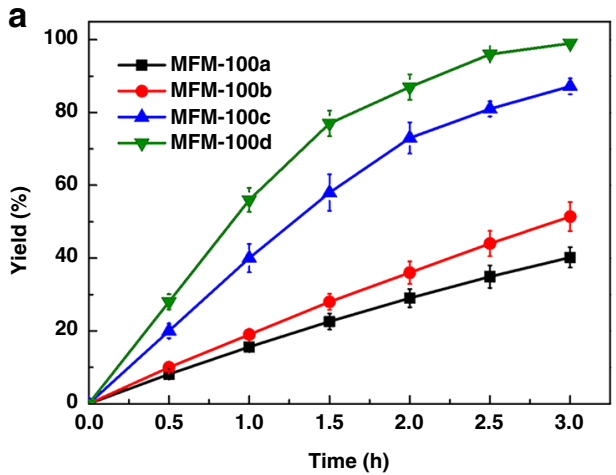

C

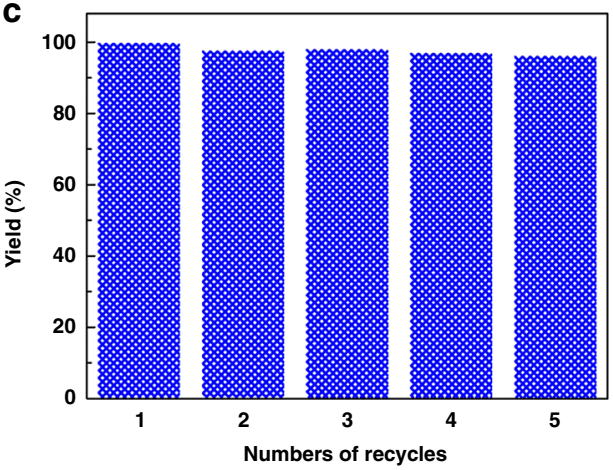

b

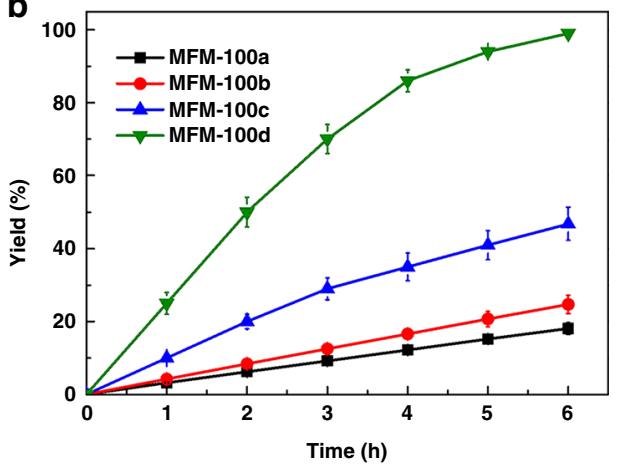

d

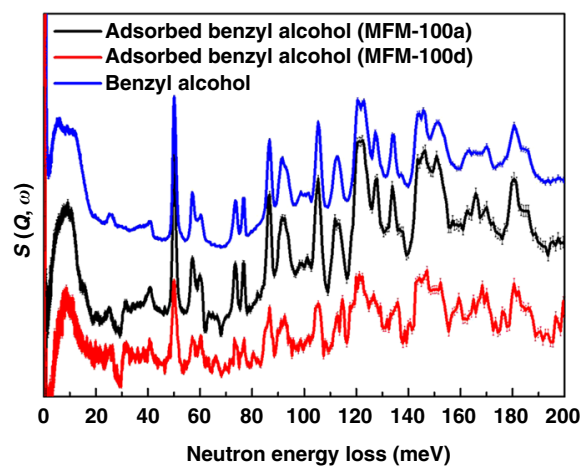

Fig. 4 Time-dependent yields of aldehydes from selected substrates catalysed over MFM-100. Yields of oxidation of a benzyl alcohol (4.3 $\left.\times 7.5 \AA^{2}\right)$ and b salicin $\left(7.0 \times 9.5 \AA^{2}\right)$. c Reusability of MFM-100d (entry 4 in Table 2). d Comparison of INS spectra of solid-state and adsorbed benzyl alcohol in MFM100a and MFM-100d. The error bars in $\mathbf{a}$ and $\mathbf{b}$ were obtained by repeating each reaction three times

catalytic performance observed for MFM-100d. The method developed here paves a new and easy-scalable pathway to synthesise mesoporous, defect MOFs as efficient catalysts for alcohol oxidation.

\section{Methods}

Solvothermal synthesis of MOFs. MFM-100a was synthesised following the reported method ${ }^{27,28}$. In a typical experiment, $\mathrm{Cu}\left(\mathrm{NO}_{3}\right)_{2} \cdot 2 \cdot 5 \mathrm{H}_{2} \mathrm{O}(0.20 \mathrm{~g}, 0.86 \mathrm{mmol})$ and biphenyl-3,3',5,5'-tetracarboxylic acid $\left(\mathrm{H}_{4} \mathrm{~L}\right)(0.10 \mathrm{~g}, 0.30 \mathrm{mmol})$ were mixed in $\mathrm{DMF} /$ dioxane $/ \mathrm{H}_{2} \mathrm{O}(2: 1: 1 \mathrm{v} / \mathrm{v} / \mathrm{v})(75 \mathrm{~mL})$ containing hydrochloric acid solution (37\%) $(0.5 \mathrm{~mL})$. The solution was placed in an autoclave and heated at $80^{\circ} \mathrm{C}$ for 3 days. The as-synthesised material was collected via filtration and washed with hot DMF and acetone, each for five times, and dried at $60^{\circ} \mathrm{C}$. HKUST-1a and MOF-2a were synthesised similarly in autoclaves at 60 and $110^{\circ} \mathrm{C}$, respectively ${ }^{32}$.

Electrosynthesis of MOFs. All syntheses were performed on a CHI660E electrochemical workstation. In a typical experiment, $\mathrm{H}_{4} \mathrm{~L}(0.05 \mathrm{~g}, 0.15 \mathrm{mmol})$ and a fixed amount $(1,10$ and $50 \mathrm{wt} \%$ for MFM-100b, MFM-100c, and MFM-100d, respectively) of IL were mixed in a stirred solution of DMF/dioxane/ $\mathrm{H}_{2} \mathrm{O}(2: 1: 1 \mathrm{v} /$ v/v) $(75 \mathrm{~mL})$ containing hydrochloric acid solution $(37 \%)(0.5 \mathrm{~mL})$ at $80^{\circ} \mathrm{C}$ for MFM- $100 \mathrm{~b}$ and at $25^{\circ} \mathrm{C}$ for MFM-100c and MFM- $100 \mathrm{~d}$ for $10 \mathrm{~min}$. The resultant clear solution was used as the electrolyte. Copper foils $\left(2 \times 1 \mathrm{~cm}^{2}\right)$ were used as both cathode and anode, which were immersed into the electrolyte, and the electrolysis conducted under an applied potential of $8 \mathrm{~V}$ (Fig. 1e). A blue precipitate formed rapidly as the electrolysis proceeded for $100 \mathrm{~s}$. The electrolysis was continued for a further $400 \mathrm{~s}$, and the precipitate collected via filtration and washed with DMF and acetone, each for five times, and the material dried at $60^{\circ} \mathrm{C}$. Electrosynthesis of HKUST-1b and MOF-2b were carried out using the same condition as MFM-100d except using trimesic and terephthalic acid as linkers.

Material characterisations. PXRD data were collected on the X-ray diffractometer (Model D/MAX2500, Rigaka) with $\mathrm{Cu}-\mathrm{Ka}$ radiation at a scan speed of $2{ }^{\circ} \mathrm{C} / \mathrm{min}$. FTIR data were collected on a Bruker Vertex spectrometer on Beamline B22 at Diamond Light Source, while TGA measurements were performed under $\mathrm{N}_{2}$ at a heating rate of $10^{\circ} \mathrm{C} / \mathrm{min}$. Contact angle measurements between $\mathrm{MOF}$ and a water droplet were obtained using Biolin Attension goniometer, and the porosity of these materials was studied by adsorption-desorption isotherms for $\mathrm{N}_{2}$ using a Micromeritics ASAP 2020M system. Prior to the BET measurement, all samples were activated under dynamic vacuum at $130^{\circ} \mathrm{C}$ for $12 \mathrm{~h}$. The $\mathrm{BJH}$ method and the
Horvath-Kawazoe method were used for analysis of mesopores and micropores, respectively. The morphologies of the materials were characterised by SEM on a Quanta 650 and TEM on a FEI Tecnai T20.

Samples preparation and measurement of CFM. The procedure of sample preparation and measurement of CFM data was same as that reported previously ${ }^{30}$ A $10 \mathrm{mg}$ sample of MOF was immersed in furfuryl alcohol $(1 \mathrm{~mL})$ at $80^{\circ} \mathrm{C}$ for $24 \mathrm{~h}$. After this incubation step, the MOF was collected by filtration and dried. An Olympus Fluoview FV-1000 instrument was used to record fluorescence micrographs.

EPR measurements. The EPR spectra arising from solid MFM-100 samples were recorded in continuous-wave at $9 \mathrm{GHz}$ (X-band) using a Bruker Micro spectrometer at room temperature with a microwave power of $2 \mathrm{~mW}$, and the spectra reported herein were typically the average of 20 scans. The intensity of the EPR signal of different samples was normalised to the sample quantity.

INS experiments and DFT calculations. INS spectra were recorded on the TOSCA spectrometer at the ISIS Facility at the STFC Rutherford Appleton Laboratory ${ }^{41}$. TOSCA is an indirect geometry crystal analyser instrument that provides a wide dynamic range $\left(16-4000 \mathrm{~cm}^{-1}\right)$ with resolution optimised in the $50-2000 \mathrm{~cm}^{-1}$ range. In this region TOSCA has a resolution of $1.25 \%$ of the energy transfer. For background tests, samples were loaded into an in situ catalysis cell with a copper vacuum seal and connected to a gas handling system. The sample was heated at $100{ }^{\circ} \mathrm{C}$ for $12 \mathrm{~h}$ under He flow to remove any remaining trace water before the experiment. The samples were cooled to below $15 \mathrm{~K}$ by a closed cycle refrigerator cryostat for data collection ${ }^{42}$. The MOF sample $(0.7 \mathrm{~g})$ was dosed with benzyl alcohol $(0.5 \mathrm{~g})$ and sealed in the cell, which was then heated at $100^{\circ} \mathrm{C}$ for $24 \mathrm{~h}$ to reach equilibrium. Data collection was conducted in the same way as the background collection. DFT calculations were performed using CASTEP ${ }^{43}$. The generalised gradient approximation, as implemented by Perdew-Burke-Ernzerhof, was used to describe the exchange-correlation interactions. Ultra-soft pseudopotentials were employed to account for the effects of core electrons. Tkatchenko-Scheffler dispersion correction was used for van der Waals interactions ${ }^{44}$. Energy cutoff for plane-wave basis was $450 \mathrm{eV}$, and the unit cell configuration determined by XRD was used as the initial structure for the simulations. The atomic coordinates were relaxed to allow minimisation of the potential energy and the interatomic forces. The energy tolerance for the electronic structure calculations was $5 \times 10^{-10} \mathrm{eV}$, for ionic relaxation $5 \times 10^{-9} \mathrm{eV}$ and the tolerance for the interatomic forces was $1 \mathrm{meV} / \AA$. After convergence was reached, the dynamical 
matrix was obtained using the finite displacement method, from which the phonon frequencies and vibrational modes were calculated. The electronic structure calculations were performed on the $\Gamma$-point only for the MOF due to its large unit cell (crystal structure: this work), and on a $5 \times 7 \times 3$ Monkhorst-Pack mesh for benzyl alcohol (crystal structure: CCDC 725239). The supercell used for the phonon calculation was $1 \times 1 \times 1$ of the unit cell for the MOF, and $2 \times 3 \times 1$ of the unit cell for benzyl alcohol. Phonon sampling in the Brillouin zone was performed on a $3 \times$ $3 \times 2 \Gamma$-centred mesh for the MOF, and $7 \times 9 \times 5$ Monkhorst-Pack mesh for benzyl alcohol. The OClimax software was used to convert the DFT-calculated phonon results to the simulated INS spectra ${ }^{45}$.

Oxidation of alcohols. All oxidation reactions were carried out in three-necked flasks using similar protocols. The oxidation of benzyl alcohol is described here in detail. Benzyl alcohol (108 mg, $1 \mathrm{mmol})$, MOF (0.1 mmol, based on the Cu content from ICP), 0.1 equiv TEMPO and 0.2 equiv $\mathrm{K}_{2} \mathrm{CO}_{3}$ (pellets of diameter of $\sim 1 \mathrm{~mm}$ ) were loaded into $4 \mathrm{~mL}$ of $\mathrm{MeCN}$. The air in the flask was replaced by $\mathrm{O}_{2}$, and the flask immersed in an oil bath at $75^{\circ} \mathrm{C}$. The $\mathrm{O}_{2}$ at ambient pressure was supplied by a balloon of $\mathrm{O}_{2}$. After reaction, the mixture was centrifuged and the solution taken at the targeted reaction time and analysed by GC and ${ }^{1} \mathrm{H}$ NMR spectroscopy. Salicin and the corresponding aldehyde were analysed by ${ }^{1} \mathrm{H}$ NMR spectroscopy and other reactants and products analysed by gas chromatography (Supplementary Fig. 23).

Stability of the catalyst. The reaction mixture was centrifuged and the solid MOF catalyst containing $\mathrm{K}_{2} \mathrm{CO}_{3}$ was collected, washed with acetone, dried at $60^{\circ} \mathrm{C}$ for $24 \mathrm{~h}$, and re-used for the next run. Five cycles of repeated tests were conducted. For the leaching test, the reaction mixture at $50 \%$ conversion and the supernatant were checked for reactivity with fresh $\mathrm{K}_{2} \mathrm{CO}_{3}$. After the catalysis, $\mathrm{K}_{2} \mathrm{CO}_{3}$ and MFM-100d catalyst were separated from the suspension for further characterisation.

\section{Data availability}

The data that support the findings of this study are available from the corresponding authors on reasonable request.

Received: 28 April 2019 Accepted: 27 August 2019

Published online: 02 October 2019

\section{References}

1. Drake, T., Ji, P. F. \& Lin, W. B. Site isolation in metal-organic frameworks enables novel transition metal catalysis. Acc. Chem. Res. 51, 2129-2138 (2018).

2. Jiao, L., Wang, Y., Jiang, H. L. \& Xu, Q. Metal-organic frameworks as platforms for catalytic applications. Adv. Mater. 30, 1703663 (2018).

3. Ma, L. Q., Falkowski, J. M., Abney, C. \& Lin, W. A. Series of isoreticular chiral metal-organic frameworks as a tunable platform for asymmetric catalysis. Nat. Chem. 2, 838-846 (2010).

4. Li, P. et al. Hierarchically engineered mesoporous metal-organic frameworks toward cell-free immobilized enzyme systems. Chem 4, 1022-1034 (2018).

5. Li, P. et al. Bottom-up construction of a superstructure in a porous uraniumorganic crystal. Science 356, 624-627 (2017).

6. Liu, D. X., Zou, D. T., Zhu, H. L. \& Zhang, J. Y. Mesoporous metal-organic frameworks: synthetic strategies and emerging applications. Small 14, 1801454 (2018).

7. Peng, L. et al. Highly mesoporous metal-organic framework assembled in a switchable solvent. Nat. Commun. 5, 4465 (2014).

8. Kozachuk, O. et al. Multifunctional, defect-engineered metal-organic frameworks with ruthenium centers: sorption and catalytic properties. Angew. Chem. Int. Ed. 53, 7058-7062 (2014).

9. Ling, S. L. \& Slater, B. Dynamic acidity in defective UiO-66. Chem. Sci. 7, 4706-4712 (2016).

10. Yuan, S. et al. Construction of hierarchically porous metal-organic frameworks through linker labilization. Nat. Commun. 8, 15356 (2017).

11. Li, T., Kozlowski, M. T., Doud, E. A., Blakely, M. N. \& Rosi, N. L. Stepwise ligand exchange for the preparation of a family of mesoporous MOFs. J. Am. Chem. Soc. 135, 11688-11691 (2013).

12. Zhang, X., Zhang, X., Johnson, J. A., Chen, Y. S. \& Zhang, J. Highly porous zirconium metal-organic frameworks with $\beta$ - $\mathrm{UH}_{3}$-like topology based on elongated tetrahedral linkers. J. Am. Chem. Soc. 138, 8380-8383 (2016).

13. Kang, X. C. et al. One-step synthesis of highly efficient nanocatalysts on the supports with hierarchical pores using porous ionic liquid-water gel. J. Am. Chem. Soc. 136, 3768-3771 (2014).

14. Yue, Y. F., Fulvio, P. F. \& Dai, S. Hierarchical metal-organic framework hybrids: perturbation-assisted nanofusion synthesis. Acc. Chem. Res. 48, 3044-3052 (2015).
15. Guan, H. Y., LeBlanc, R. J., Xie, S. Y. \& Yue, Y. F. Recent progress in the syntheses of mesoporous metal-organic framework materials. Coord. Chem. Rev. 369, 76-90 (2018).

16. Zhang, L. L. et al. Pore-environment engineering with multiple metal sites in rare-earth porphyrinic metal-organic frameworks. Angew. Chem. Int. Ed. 57, 5095-5099 (2018).

17. Položij, M., Rubeš, M., Čejka, J. \& Nachtigall, P. Catalysis by dynamically formed defects in a metal-organic framework structure: knoevenagel reaction catalyzed by copper benzene-1,3,5-tricarboxylate. ChemCatChem $\mathbf{6}$, 2821-2824 (2014).

18. Xie, S. F., Xu, Q. C. \& Huang, X. Q. Defect-rich metal nanocrystals in catalysis. ChemCatChem 8, 480-485 (2016).

19. Fang, Z. L., Bueken, B., De Vos, D. E. \& Fischer, R. A. Defect-engineered metal-organic frameworks. Angew. Chem. Int. Ed. 54, 7234-7254 (2015).

20. Guillerm, V. et al. A series of isoreticular, highly stable, porous zirconium oxide based metal-organic frameworks. Angew. Chem. Int. Ed. 51, 9267-9271 (2012).

21. Cliffe, M. J. et al. Correlated defect nanoregions in a metal-organic framework. Nat. Commun. 5, 4176 (2014).

22. Tu, B. B. et al. Ordered vacancies and their chemistry in metal organic frameworks. J. Am. Chem. Soc. 136, 14465-14471 (2014).

23. Shearer, G. C. et al. Tuned to perfection: ironing out the defects in metalorganic framework UiO-66. Chem. Mater. 26, 4068-4071 (2014).

24. Vermoortele, F. et al. Tuning the catalytic performance of metal-organic frameworks in fine chemistry by active site engineering. J. Mater. Chem. 22, 10313-10321 (2012).

25. Ameloot, R. et al. Patterned growth of metal-organic framework coatings by electrochemical synthesis. Chem. Mater. 21, 2580-2582 (2009).

26. Li, M. Y. \& Dincă, M. Reductive electrosynthesis of crystalline metal-organic frameworks. J. Am. Chem. Soc. 133, 12926-12929 (2011).

27. Lin, $\mathrm{X}$. et al. High $\mathrm{H}_{2}$ adsorption by coordination-framework materials. Angew. Chem. Int. Ed. 45, 7358-7364 (2006).

28. Lin, $\mathrm{X}$. et al. High capacity hydrogen adsorption in $\mathrm{Cu}(\mathrm{II})$ tetracarboxylate framework materials: the role of pore size, ligand functionalization and exposed metal sites. J. Am. Chem. Soc. 131, 2159-2171 (2009).

29. EL Mkami, H. et al. EPR and magnetic studies of a novel copper metal organic framework (STAM-I). Chem. Phys. Lett. 544, 17-21 (2012).

30. Ameloot, R. et al. Three-dimensional visualization of defects formed during the synthesis of metal-organic frameworks: a fluorescence microscopy study Angew. Chem. Int. Ed. 52, 401-405 (2013).

31. Kang, X. C., Sun, X. F. \& Han, B. X. Synthesis of functional nanomaterials in ionic liquids. Adv. Mater. 28, 1011-1030 (2016)

32. Kim, H. K. et al. A chemical route to activation of open metal sites in the copper-based metal-organic framework materials HKUST-1 and Cu-MOF-2. J. Am. Chem. Soc. 137, 10009-10015 (2015).

33. Enache, D. I. et al. Solvent-free oxidation of primary alcohols to aldehydes using $\mathrm{Au}-\mathrm{Pd} / \mathrm{TiO}_{2}$ catalysts. Science 311, 362-365 (2006).

34. Hoover, J. M. \& Stahl, S. S. Highly practical copper(I)/TEMPO catalyst system for chemoselective aerobic oxidation of primary alcohols. J. Am. Chem. Soc. 133, 16901-16910 (2011)

35. Ryland, B. L., McCann, S. D., Brunold, T. C. \& Stahl, S. S. Mechanism of alcohol oxidation mediated by copper(II) and nitroxyl radicals. J. Am. Chem. Soc. 136, 12166-12173 (2014).

36. Ryland, B. L. \& Stahl, S. S. Practical aerobic oxidations of alcohols and amines with homogeneous copper/TEMPO and related catalyst systems. Angew. Chem. Int. Ed. 53, 8824-8838 (2014).

37. Xu, B. R., Lumb, J. P. \& Arndtsen, B. A. A TEMPO-free copper-catalyzed aerobic oxidation of alcohols. Angew. Chem. Int. Ed. 54, 4208-4211 (2015).

38. Stepanova, E. V., Belyanin, M. L. \& Filimonov, V. D. Synthesis of acyl derivatives of salicin, salirepin, and arbutin. Carbohyd. Res. 388, 105-111 (2014).

39. Chen, S. C. et al. Highly selective aerobic oxidation of alcohols to aldehydes over a new $\mathrm{Cu}(\mathrm{II})$-based metal-organic framework with mixed linkers. Catal. Commun. 95, 6-11 (2017).

40. Xue, Z. M. et al. Poly(ethylene glycol) stabilized mesoporous metal-organic framework nanocrystals: efficient and durable catalysts for the oxidation of benzyl alcohol. Chemphyschem 15, 85-89 (2014).

41. Xia, Q. N. et al. Direct hydrodeoxygenation of raw woody biomass into liquid alkanes. Nat. Commun. 7, 11162 (2016).

42. Shao, Y. et al. Selective production of arenes via direct lignin upgrading over a niobium-based catalyst. Nat. Commun. 8, 16104 (2017).

43. Clark, S. J. et al. First principles methods using CASTEP. Z. Kristallogr. 220, 567-570 (2005)

44. McNellis, E. R., Meyer, J. \& Reuter, K. Azobenzene at coinage metal surfaces: role of dispersive van der Waals interactions. Phys. Rev. B 80, 205414 (2009).

45. Ramirez-Cuesta, A. J. aCLIMAX 4.0.1, The new version of the software for analyzing and interpreting INS spectra. Comput. Phys. Commun. 157, 226-238 (2004) 


\section{Acknowledgements}

We thank EPSRC (EP/I011870), ERC (AdG 742041), the Royal Society, University of Manchester and Institute of Chemistry, Chinese Academy of Science for funding. X.K. is supported by a Royal Society Newton International Fellowship. We are especially grateful to Diamond Light Source and STFC/ISIS Facility for access to the Beamlines B22 and TOSCA, respectively. The computing resources were made available through the VirtuES and the ICE-MAN projects, funded by Laboratory Directed Research and Development programme at ORNL. KL and JL acknowledge the China Scholarship Council for support of PhD studentships.

\section{Author contributions}

X.K., K.L. and L. Li: syntheses and characterisation of MOF samples. X.K. and J.L.: collection of FTIR and INS data. X.K. and L.K.: catalysis tests. B.W. and R.A.W.D.: collection and analysis of EPR data. L. Liu: CFM measurements. Y.C., S.R. and A.J.R.-C.: analysis of INS data and DFT calculations. M.D.F.: FTIR data analysis. B.H., S.Y. and M.S.: overall direction, design and supervision of the project. X.K., S.Y., and M.S.: preparation of the paper with all authors.

\section{Additional information}

Supplementary Information accompanies this paper at https://doi.org/10.1038/s41467019-12268-5.

Competing interests: The authors declare no competing interests.
Reprints and permission information is available online at http://npg.nature.com/ reprintsandpermissions/

Peer review information Nature Communications thanks Peng Li and the other, anonymous, reviewer(s) for their contribution to the peer review of this work.

Publisher's note Springer Nature remains neutral with regard to jurisdictional claims in published maps and institutional affiliations.

(c) (i) Open Access This article is licensed under a Creative Commons Attribution 4.0 International License, which permits use, sharing, adaptation, distribution and reproduction in any medium or format, as long as you give appropriate credit to the original author(s) and the source, provide a link to the Creative Commons license, and indicate if changes were made. The images or other third party material in this article are included in the article's Creative Commons license, unless indicated otherwise in a credit line to the material. If material is not included in the article's Creative Commons license and your intended use is not permitted by statutory regulation or exceeds the permitted use, you will need to obtain permission directly from the copyright holder. To view a copy of this license, visit http://creativecommons.org/ licenses/by/4.0/.

(c) The Author(s) 2019 